\title{
Rating competitors before tournament starts: how it's affecting team progression in a soccer tournament
}

\begin{abstract}
In professional sporting events, rating competitors before tournament start is a well-known approach to distinguish the favorite team and the weaker teams. Various methodologies are used to rate competitors. In this paper, we explore four ways to rate competitors; least squares rating, maximum likelihood strength ratio, standing points in large round robin simulation and previous league rank position. The tournament metric we used to evaluate different types of rating approach is tournament outcome characteristics measure. The tournament outcome characteristics measure is defined by the probability that a particular team in the top $100 \mathrm{q}$ pre-tournament rank percentile progress beyond round $\mathrm{R}$, for all $\mathrm{q}$ and $\mathrm{R}$. Based on simulation result, we found that different rating approach produces different effect to the team. Our simulation result shows that from eight teams participate in knockout standard seeding, Perak has highest probability to win for tournament that use the least squares rating approach, PKNS has highest probability to win using the maximum likelihood strength ratio and the large round robin simulation approach, while Perak has the highest probability to win a tournament using previous league season approach.
\end{abstract}

Keyword: Rating competitors; Simulations studies; Soccer 